\title{
Analyse du fonctionnement des groupes axiaux de grandes dimensions
}

\section{An operational analysis of large axial units}

\author{
S. X. CASACCI \\ INGÉNIEUR-DOCTEUR \\ CHEF DU DÉPARTEMENT 《TURBINES 》 \\ AUX ÉTABLISSEMENTS NEYRPIC, GRENOBLE
}

PAR

ET

Rappel des problèmes de génie civil, d'hydraulique et d'hydromécanique liés à la tenue des parties tournantes, $\dot{a}$ la rigidite des supports et $\dot{a}$ la commande des pales, qui conduisirent $\dot{a}$ envisager successivement, pour l'équipement de la centrale marémotrice de la Rance, des groupes axiaux de grandes dimensions $\dot{a}$ pales retournables en marche, des groupes du type "bulbe aval» réalisés pour les machines de Cambeyrac et d'Argentat, enfin des groupes «bulbe amont» réalisés à la centrale de Beaumont-Monteux et à la station expérimentale de Saint-Malo.

Problèmes spéciaux posés pour la réalisation de ce groupe «bulbe amont》 de grandes dimensions (roue de diamètre $5,8 \mathrm{~m}$ ). Solution de quelques-uns d'entre eux:

- Divers fonctionnements hydrauliques permanents ou transitoires:

- Tenue mécanique des parties tournantes;

- Rigidité des pièces supports et liaisons an génie civil;

- Lutte contre les vibrations des parties tournantes et des pièces supports.

Analyse des possibilités d'application de ces conceptions $\dot{a}$ des machines axiales plus puissantes.

Le fonctionnement du groupe expérimental de Saint-Malo a montré que l'on peut envisager avec sûreté l'équipement des usines marémotrices. Des nombreux problèmes que posent l'équipement et l'exploitation de ces usines, nous n'examinerons ici que ceux soulevés par la construction des groupes axiaux de grandes dimensions et ceux liés aux différents fonctionnements hydrauliques des machines marémotrices.

\author{
M. KAMMERLOCHER \\ ANCIEN CHEF DU SERVICE ÉleCtRoMj́canique \\ DE LA RÉGION D'ÉQUIPEMENT «MARÉMOTRICE》A E.D.F. \\ INGÉNIEUR A LA SOCIÉTÉ INDATOM, PARIS
}

Dans une installation comportant un groupe axial, la machine elle-même constitue une partie du conduit hydraulique. L'alternateur qui baigne dans le fluide moteur et les parties fixes de la turbine qui servent de supports, ne doivent donc pas réduire exagérément la section du conduit hydraulique. Les alternateurs accouplés à ces turbines axiales, vu leur faible diamètre, ont des longueurs de fer relativement importantes; 
la recherche de bonnes performances oblige d'autre part à réduire leur entrefer. Le bon fonctionnement de ces machines électriques à grande longueur de fer et à faible entrefer exige que les déformations des parties tournantes et des pièces supports soient faibles. Les parties fixes de la turbine qui constituent les supports doivent donc être très rigides sans toutefois présenter une grande obstruction au passage du fluide moteur. Les déformations de pièces de dimensions importantes, comme la carcasse de l'alternateur, ne restent limitées dans le temps que si les liaisons des pièces supports au béton ont peu d'influence sur ces déformations. Les liaisons multiples et de rigidité comparable sont donc à éviter, car il risque de se produire des déformations dues au fluage du béton et aux champs de température. De plus, les appuis multiples dissymétriques peuvent provoquer des déformations locales importantes tout en réduisant les déformations moyennes.

Les fonctionnements hydrauliques multiples nécessités par l'exploitation des usines marémotrices rendent encore plus impératives la limitation des déformations et la lutte contre les vibrations.

Toutes ces conditions ont conduit à la conception du groupe de Saint-Malo qui ne comporte qu'une seule liaison rigide au béton au droit de la fixation de l'avant-distributeur et qu'un seul appui rigide : l'avant-distributeur. L'alternateur et le cône support du palier turbine sont boulonnés en porte-á-faux de part et d'autre de l'avantdistributeur. Des tirants précontraints, fixés à l'une de leurs extrémités dans le béton et à l'autre sur un anneau situé entre la calotte et la carcasse de l'alternateur permettent d'étouffer les vibrations de l'ensemble du groupe par rapport au centre de l'avant-distributeur.

Pour réaliser les différents régimes de fonctionnement hydrauliques, le groupe expérimental de Cambeyrac comporte des pales retournables dont la course angulaire maximale atteint $220^{\circ}$. Le mécanisme de manœuvre des pales, compliqué et lourd, accroît les difficultés de construction des groupes bulbes avec roue en porte-à-faux. Son adoption à Saint-Malo nous aurait probablement obligés à placer dans le conduit hydraulique un croisillon supplémentaire permettant de supporter la roue sur deux paliers. Nous avons jugé préférable de réaliser des pales de tracé spécial ayant une course angulaire maximale de $45^{\circ}$ dont la manœuvre peut être assurée par un mécanisme classique du type bielle et manivelle. Nous avons pu ainsi conserver à la machine sa conception mécanique simple comportant une roue en porte-à-faux. Signalons de plus que des pales non retournables présentent le grand avantage de permettre le passage continu d'un régime de fonctionnement à l'autre.

\section{I. - CONCEPTION DU GROUPE}

Le groupe de Saint-Malo (fig. 1) comporte un seul appui : l'avant-distributeur, constitué de huit avant-directrices pleines de $120 \mathrm{~mm}$ d'épaisseur et quatre creuses de $250 \mathrm{~mm}$ de maîtrecouple. La largeur des avant-directrices est de $900 \mathrm{~mm}$. Le diamètre moyen de la paroi extérieure atteint $8700 \mathrm{~mm}$, celui de la paroi intérieure $4600 \mathrm{~mm}$. De part et d'autre de l'anneau intérieur de l'avant-distributeur sont boulonnés le cône support du palier turbine et la carcasse de l'alternateur. Des tirants précontraints sont fixés à l'une de leurs extrémités dans le béton et à l'autre sur un anneau de renfort situé entre la calotte et la carcasse de l'alternateur. Le bras qui permet d'accéder à l'intérieur de la calotte n'est pas lié rigidement au béton et ne constitue donc pas un support du groupe.

Les tirants précontraints, disposés suivant des rayons faisant un angle de $45^{\circ}$ avec les axes rendent négligeable toute déformation autour d'axes situés dans le plan moyen de l'avantdistributeur. Les basculements de l'ensemble du moulinet autour de ces axes doivent en effet être très faibles pour que tout contact entre la roue et la ceinture soit évité, même pour des fonctionnements hydrauliques très perturbés tels que les emballements en turbine inversée. 


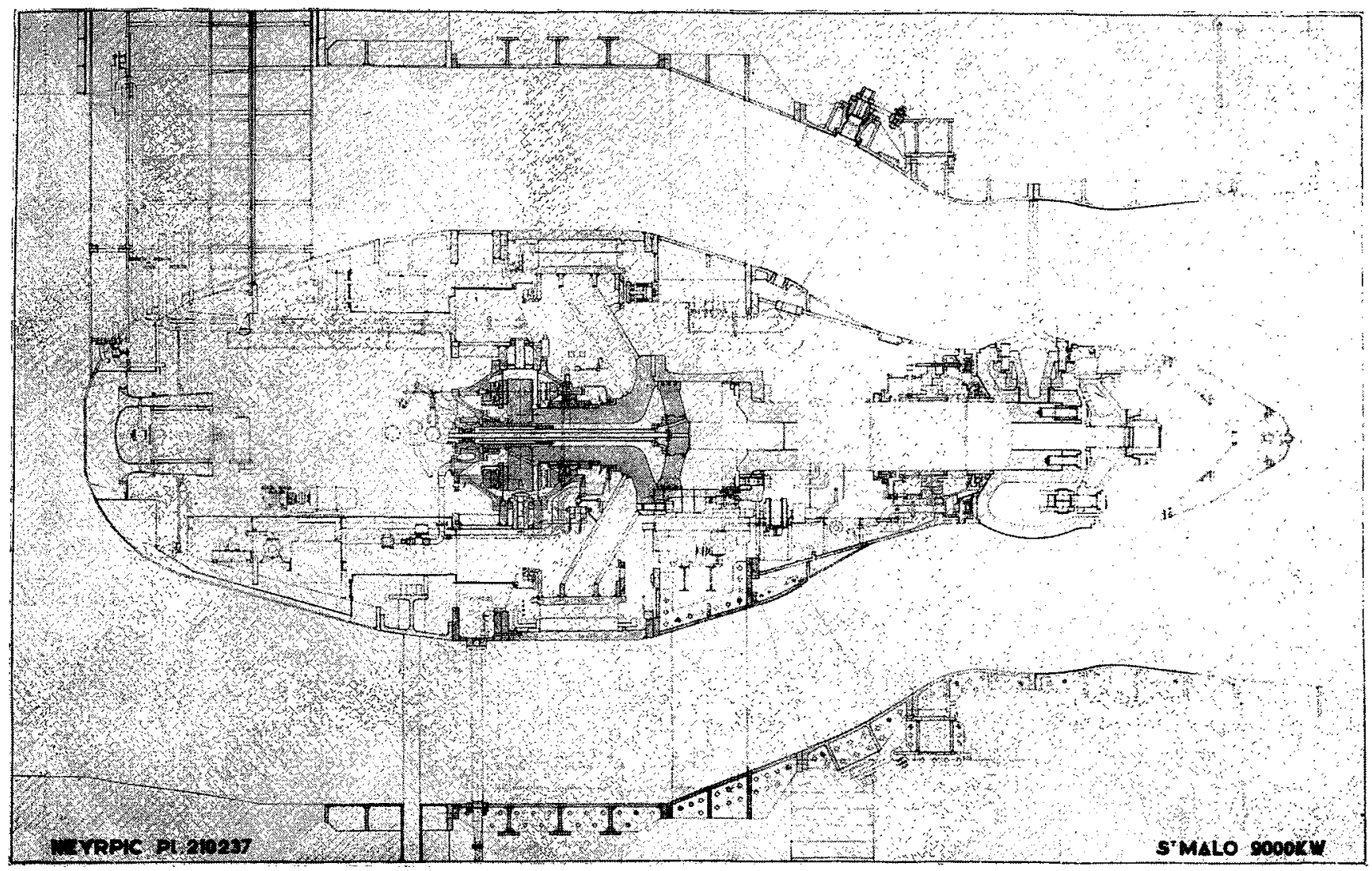

Frg. 1

\section{II. - RIGIDITE ET FREQUENCE PROPRE DU GROUPE POUR DES DEPLACEMENTS DE TRANSLATION LE LONG DE L'AXE DE LA MACHINE}

Le poids de l'ensemble du moulinet atteint environ 300 tonnes. Si l'on suppose les avantdirectrices parfaitement encastrées dans les anneaux extérieur et intérieur de l'avant-distributeur, il faut une force de 2000 tonnes pour provoquer un déplacement axial de 1 millimètre.

La fréquence propre dans l'air est donc d'environ $40 \mathrm{~Hz}$. En toute rigueur, il faudrait tenir compte de la masse répartie des avant-directrices. Le calcul montre que la fréquence propre reste très voisine de $40 \mathrm{~Hz}$, car la masse de l'ensemble des avant-directrices est négligeable par rapport à la masse totale du moulinet.

La fréquence propre de l'ensemble constitué par le moulinet fixé aux avant-directrices est bien entendu fonction du milieu ambiant. Si l'on assimile le bulbe à un ellipsoïde de révolution dont l'axe de révolution est le double de l'autre, la masse associée à prendre en compte représente environ les $2 / 10^{\text {es }}$ de la masse d'eau dépla- cée par le bulbe. Cette valeur correspond au cas d'un ellipsoïde vibrant dans un milieu fluide parfait infini et initialement au repos. Le poids d'eau déplacé par le bulbe atteint environ 150 tonnes. La fréquence propre dans l'eau se situe donc aux environs de $39 \mathrm{~Hz}$.

En fait, le bulbe oscille axialement dans un conduit où s'écoule de l'eau; il faudrait donc analyser l'influence de la présence de la paroi d'une part, et de la vitesse d'écoulement d'autre part. Notons de plus que pour la grille d'aubes de la roue une certaine masse d'eau associée intervient. Ces diverses influences, relativement faibles, n'entraînent pas une diminution sensible de la fréquence propre, qu'on peut donc supposer voisine de $40 \mathrm{~Hz}$. Les forces excitatrices d'origine hydraulique, de fréquences bien inférieures à $40 \mathrm{~Hz}$, ne peuvent donc pas engendrer des vibrations axiales dangereuses. Nous n'avons en fait décelé aucune vibration axiale sensible quels que soient les régimes hydrauliques. 


\section{III. - RIGIDITE ET FREQUENCE PROPRE DU GROUPE POUR DES ROTATIONS AUTOUR D'AXES PERPENDICULAIRES \\ A L'AXE DE LA MACHINE ET PASSANT PAR LE CENTRE DE L'AVANT-DISTRIBUTEUR}

Avant la mise en eau du groupe, toute une série d'essais a été effectuée pour mesurer le basculement du groupe autour d'un axe horizontal, perpendiculaire à l'axe du groupe et situé dans le plan moyen de l'avant-distributeur. Pour mesurer la raideur du groupe, nous avons appliqué des couples de basculement :

- à l'aide des tirants supérieurs de mise en tension,

- à l'aide de vérins agissant sur l'ogive,

- en supprimant la charge due au poids de la roue.

Toutes ces mesures ont donné des résultats comparables et ont montré que le groupe tourne en bloc autour d'un axe horizontal, perpendiculaire à l'axe de la machine et situé approximalivement dans le plan moyen de l'avant-distributeur.

Pour les premiers essais, les tirants n'étaient pas sous tension. Dans ces conditions, une force verticale de 100 tonnes située dans le plan moyen des tirants provoque un déplacement vertical de $33 / 100^{\text {es }}$ de $\mathrm{mm}$ environ le long de la ligne d'action de la force. Un couple de $1 \mathrm{t} . \mathrm{m}$ provoque une déformation angulaire de l'ordre de $2,8 \cdot 10^{-7}$ radian.

Après mise en précontrainte des tirants à 70 tonnes, nous avons mesuré le basculement du groupe sous l'action d'un effort vertical appliqué par un vérin. A un couple de $1 \mathrm{t}$. $\mathrm{m}$ ne correspond plus alors qu'une déformation angulaire de $7 \cdot 10^{-8}$ radian. La présence des tirants quadruple donc la rigidité du groupe.

L'inertie du moulinet par rapport à l'axe de basculement est de l'ordre de :

$$
\mathrm{I}=2,8 \cdot 10^{5} \mathrm{~kg} \mathrm{~m} \mathrm{~s}{ }^{2}
$$

La fréquence propre correspondant à la rigidité $\mathrm{du}$ groupe sans les tirants est donc voisine de $18 \mathrm{~Hz}$; elle passe à $36 \mathrm{~Hz}$ lorsque l'on met les tirants en tension. Cette fréquence ne risque donc pas d'être excitée par des forces perturbatrices d'origine hydraulique. Nous n'avons d'ailleurs pas constaté de vibration du groupe autour d'axes perpendiculaires à l'axe de l'arbre et situés dans le plan moyen du distributeur et ce, quels que soient les régimes de fonctionnement.

\section{IV. - RIGIDITE ET FREQUENCE PROPRE DE TORSION DE L'AVANT-DISTRIBUTEUR AUTOUR DE L'AXE DU GROUPE}

L'inertie des parties fixes du moulinet par rapport à l'axe du groupe atteint environ :

$$
\mathrm{I}_{f}=5.10^{4} \mathrm{~kg} \mathrm{~m} \mathrm{~s}
$$

L'inertie des parties tournantes est de l'ordre de :

$$
\mathrm{I}_{m}=2,5 \cdot 10^{4} \mathrm{~kg} \mathrm{~m} \mathrm{~s} \mathrm{~m}^{2}
$$

Un couple de $300 \mathrm{t} . \mathrm{m}$ dont l'axe se confond avec celui de la machine provoque une déformation tangentielle de $1 \mathrm{~mm}$ sur la paroi extérieure de l'anneau intérieur de l'avant-distributeur. Précisons que le couple nominal de la machine dépasse légèrement $100 \mathrm{t} . \mathrm{m}$.

La rigidité à la torsion de l'avant-distributeur s'exprime donc par :

$$
\mathrm{K}=7,5 \cdot 10^{8} \mathrm{~m} \mathrm{~kg}
$$

Si l'on suppose que seules les parties fixes oscillent, la fréquence propre dépasse $19 \mathrm{~Hz}$; si, par contre, les parties fixes et mobiles, couplées par les forces magnétiques, tournent en bloc, la fréquence propre descend aux environs de $16 \mathrm{~Hz}$.

Ces valeurs nous semblaient assez élevées par rapport aux fréquences possibles des pulsations de couple hydraulique de la machine. En fait, des vibrations de torsion, d'amplitudes non négligeables, apparaissent lors des démarrages en moteur asynchrone et ce, que le moteur tournât dans l'air en ventilateur ou dans l'eau en moteur.

Pendant les fonctionnements dans l'air, les vibrations de torsion de l'avant-distributeur ètaient mesurées :

- sur l'avant-distributeur lui-même, à l'encastrement des avant-directrices sur l'anneau intérieur, à l'aide d'un vibromètre Ascania;

- au droit de la bride de fixation du bras d'accès sur la calotte, au moyen d'un comparateur potentiométrique, 

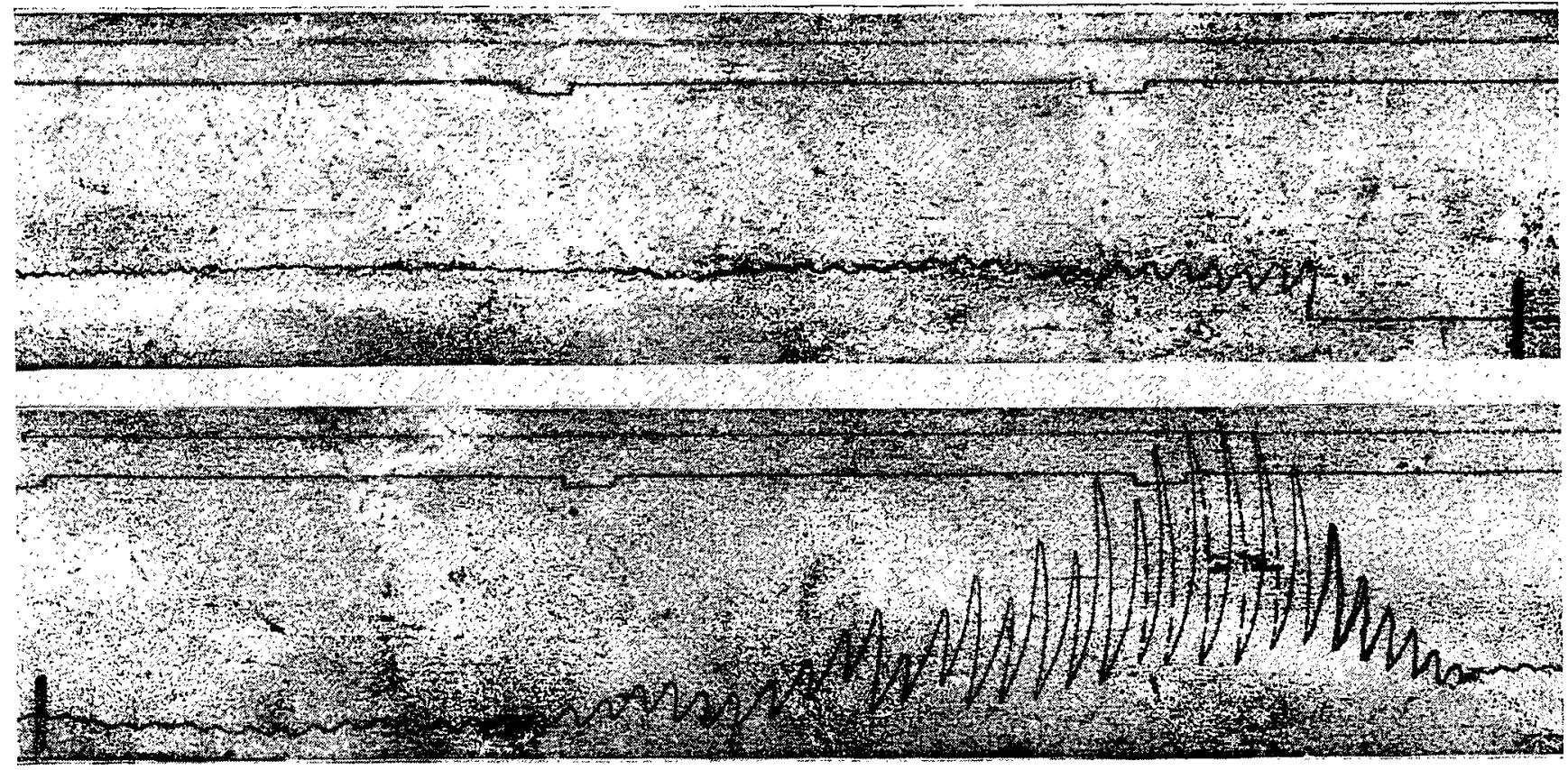

FIG. 2

Déplacements d'une A-D à l'encastrement sur l'anneau intérieur de l'avant-distributeur (amplification 40 environ).

Démarrage dans l'air en moteur asynchrone.

- au droit des paliers, avec des capteurs à variation de reluctance.

Pour les fonctionnements en eau, seules les deux dernières mesures étaient alors possibles.

\section{IV.1. Démarrage en moteur asynchrone dans l'air.}

L'enregistrement (fig. 2) se rapporte à un démarrage en moteur asynchrone dans l'air; il donne les déplacements tangentiels de l'avantdistributeur en fonction du temps. L'application brutale du couple au démarrage engendre une oscillation de torsion de fréquence voisine de $17 \mathrm{~Hz}$. L'amplitude maximale ne correspond qu'à une faible partie du couple maximal de la machine. C'est le transitoire de l'énorme accéléromètre que constitue le moulinet fixé aux avantdirectrices. On constate sur l'enregistrement, d'une part que la période propre de torsion de l'avant-distributeur est de l'ordre de $17 \mathrm{~Hz}$, d'autre part que cet accéléromètre est très peu amorti dans l'air.

La vitesse croissant, au voisinage de $73 \mathrm{tr} / \mathrm{mn}$ se produisent des vibrations de torsion d'amplitude maximale beaucoup plus élevée et de fré-
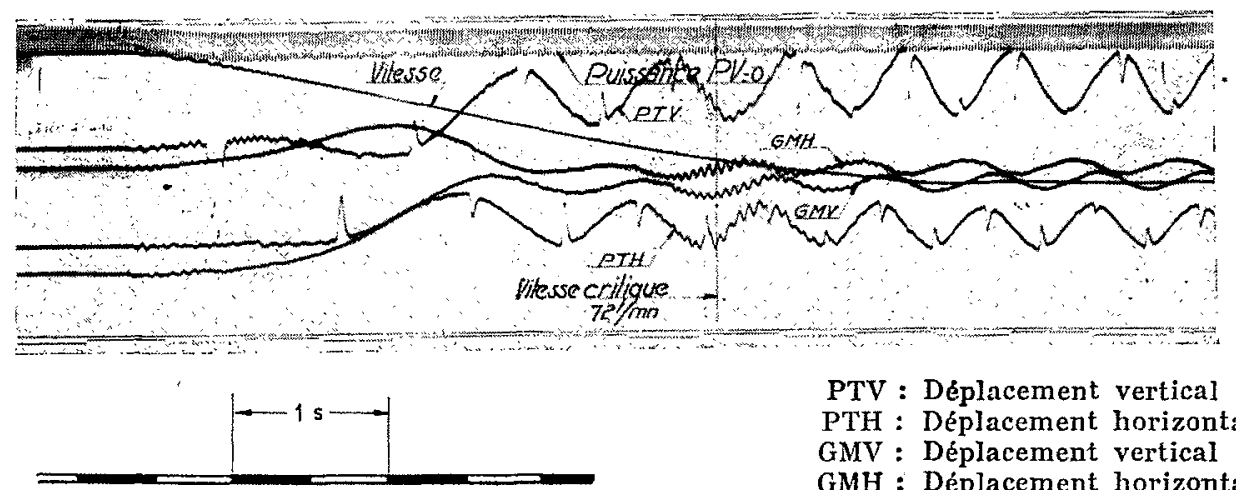

PTV : Déplacement vertical PTH : Déplacement horizontal GMV : Déplacement vertical GMH : Déplacement horizontal $\mathrm{C}_{3}$ : Déplacement tangentiel du stator.
Fic. 3

Saint-Malo.

Groupe marémoteur de $9000 \mathrm{~kW}$ sous $6 \mathrm{~m}$.

Potation dans l'air.

Démarrage en asynchrone. 
FIf, 4

Saint-Malo

Groupe marémoteur de $9000 \mathrm{~kW}$

Rotation dans l'air.

sous $6 \mathrm{~m}$.

Reprise en moteur $\dot{2} 62 \mathrm{tr} / \mathrm{mn}$.

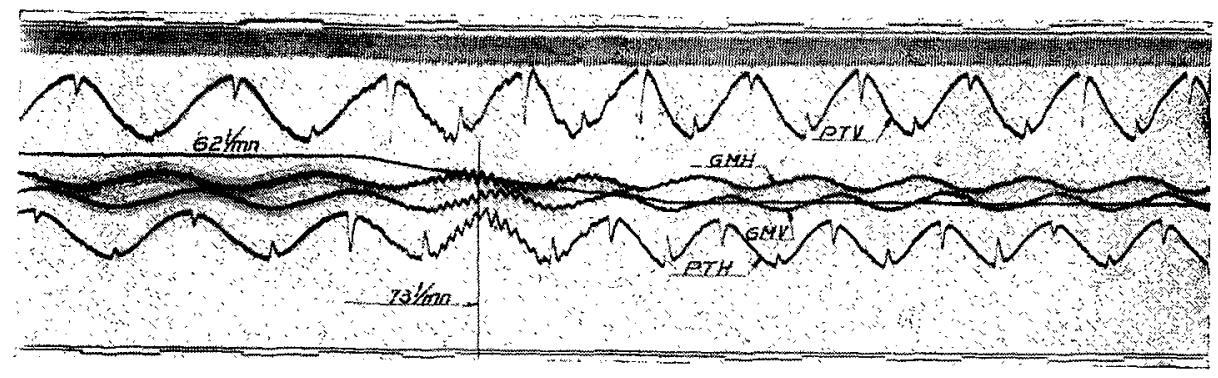

PTV : Déplacement vertical

PTH : Déplacement horizontal

GMV : Déplacement vertical

GMH : Déplacement horizontal

C. Déplacement tangentiel du stator.

Palier turbine
Grain mobile . quence toujours voisine de $17 \mathrm{~Hz}$. Ces oscillations disparaissent lentement lorsque la vitesse dépasse les $73 \mathrm{tr} / \mathrm{mn}$. A la vitesse normale, on ne décèle plus aucune vibration.

Toujours dans l'air, lors d'un déclenchement, la vitesse décroît lentement et l'on ne constate aucune oscillation de torsion, même au voisinage de $73 \mathrm{tr} / \mathrm{mn}$, pendant toute la durée du ralentissement.

Les enregistrements (fig. 3) correspondent aussi à un démarrage en moteur asynchrone dans l'air.

On retrouve les mêmes vibrations de torsion au moment de l'application brutale du couple et au voisinage de $72 \mathrm{tr} / \mathrm{mn}$.

Avant le démarrage, une injection d'huile sous pression crée un film hydrostatique épais. La courbe PTV donne les déplacements verticaux du palier turbine. L'enregistrement montre que la mise en vitesse provoque un accroissement du film atteignant $12 / 100^{\mathrm{es}}$ de $\mathrm{mm}$.

Les imperfections d'usinage et de montage mésurées sur l'arbre lui-même et non sur la soie du palier ne dépassent pas 6 à $7 / 100^{\mathrm{es}}$ de $\mathrm{mm}$, valeur très faible pour une machine de ces dimensions. La sinusoïde de grande période cor- respond à ces défauts. Les tops de cette sinusoïde repèrent le passage d'une génératrice de l'arbre devant les deux capteurs.

Les vibrations de torsion se manifestent au voisinage de $72 \mathrm{tr} / \mathrm{mn}$ sur les déplacements verticaux et horizontaux du palier turbine et du grain mobile de la butée. La fréquence de ces vibrations est d'environ $17 \mathrm{~Hz}$.

La figure 4 se rapporte à des enregistrements effectués lors d'une reprise en moteur asynchrone après un ralentissement jusqu'à $62 \mathrm{tr} /$ mn. Des vibrations de torsion de fréquence $17 \mathrm{~Hz}$ apparaissent toujours vers $73 \mathrm{tr} / \mathrm{mn}$. Si la reprise s'effectue vers $80 \mathrm{tr} / \mathrm{mn}$, on ne constate par contre aucune vibration.

\section{IV.2. Démarrages et fonctionnement en pompe inversée.}

Les enregistrements (fig. 5) sont relatifs à un démarrage électrique en pompe inversée. Nous n'avons pas pu à l'époque mesurer directement les vibrations tangentielles sur l'avant-distributeur lui-même. Nous les avons détectées au droit
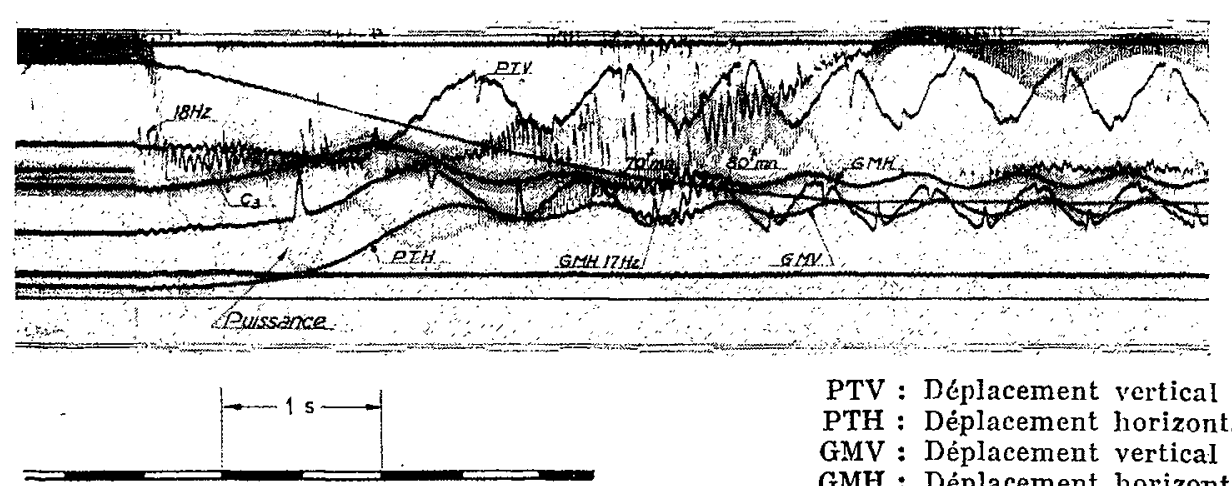

Fig. 5

Saint-Malo.

Groupe marémoteur de $9000 \mathrm{~kW}$ sous $6 \mathrm{~m}$.

Fonctionnement

en pompe inversée.

Démarrage

en moteur asynchrone.

PTV : Déplacement vertical

PTH : Déplacement horizontal

GMV : Déplacement vertical

GMH : Déplacement horizontal

$C_{3}$ : Déplacement tangentiel du stator.
Palier turbine

Grain mobile 


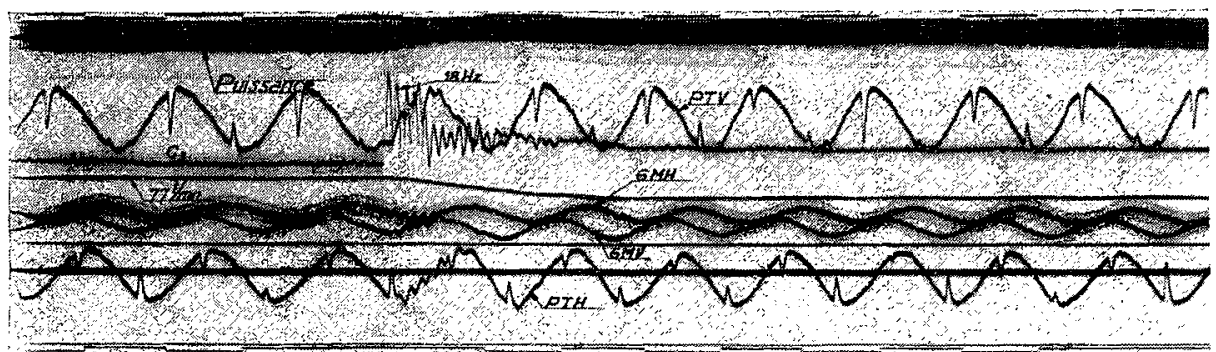

FIG. 6

Saint-Malo. Groupe marémoteur de $9000 \mathrm{hW}$ sous $6 \mathrm{~m}$. Couplage

en asynchrone à $77 \mathrm{tr} / \mathrm{mn}$ après démarrage en turbine inversée.

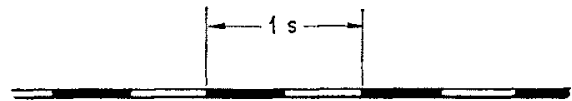

PTV : Déplacement vertical PTH : Déplacement horizontal GMV : Déplacement vertical GMH : Déplacement horizontal

$C_{s}$ : Déplacement tangentiel du stator.

des paliers et à la liaison du bras d'accès et de la calotte.

On retrouve pour les démarrages en pompe les mêmes vibrations de torsion que pour les démarrages dans l'air. Au voisinage de $73 \mathrm{tr} / \mathrm{mn}$, les amplitudes maximales à la fréquence de $17 \mathrm{~Hz}$ atteignent et même dépassent le couple nominal de la machine.

Les enregistrements (fig. 6) se rapportent à un couplage à $77 \mathrm{tr} / \mathrm{mn}$ en moteur asynchrone après une marche en turbine inversée. Des vibrations de torsion de fréquence 17 à $18 \mathrm{~Hz}$ apparaissent lors du couplage. Elles s'amortissent rapidement dès que la vitesse augmente. Les amplitudes maximales sont plus faibles que lorsqu'on couple à des vitesses inférieures à $73 \mathrm{tr}^{\circ} / \mathrm{mn}$.

Pour un couplage à $82,5 \mathrm{tr} / \mathrm{mn}$, on ne décèle aucune vibration.

Si l'on couple à $80 \mathrm{tr} / \mathrm{mn}$, on constate de légères vibrations. Pour les couplages à des vitesses inférieures à $77 \mathrm{tr} / \mathrm{mn}$, les amplitudes des vibrations de torsion deviennent relativement importantes.

A la vitesse normale, $88 \mathrm{tr} / \mathrm{mn}$, on ne mesure aucune vibration quelle que soit la puissance absorbée par la pompe.

\section{IV.3. Démarrages et fonctionnements en tur- bine inversée.}

Lors des multiples démarrages et disjonctions effectués aucune vibration nuisible n'a été constatée, même au voisinage de $73 \mathrm{tr} / \mathrm{mn}$.

Les régimes permanents les plus perturbés correspondent à de faibles angles d'inclinaison des pales. La figure 7 se rapporte à un fonctionnement en turbine inversée, l'inclinaison varie de 0 à $-3^{\circ}$. Les enregistrements des déplacements de l'arbre dans les paliers montrent qu'à la sinusoïde correspondant aux défauts mécaniques de la ligne d'arbre se superpose une oscillation parasite de fréquence assez variable et d'amplitude ne dépassant pas 2 à $3 / 100^{\mathrm{es}} \mathrm{de} \mathrm{mm}$.

Lors des dévirages en turbine inversée, aucune vibration notable n'a été constatée et ce, quel que soit l'angle d'inclinaison des pales au moment de la disjonction. Les courbes de la figure 8 donnent les variations en fonction du temps :

- de la vitesse,

- des pressions au point haut de la ceinture,

- des pressions au point haut du cône turbine,

FIG. 7

Saint-Malo.

Groupe marémoteur de $9000 \mathrm{~kW}$ sous $6 \mathrm{~m}$.

Marche en pompe inversée;

régime hydraulique perturbé.

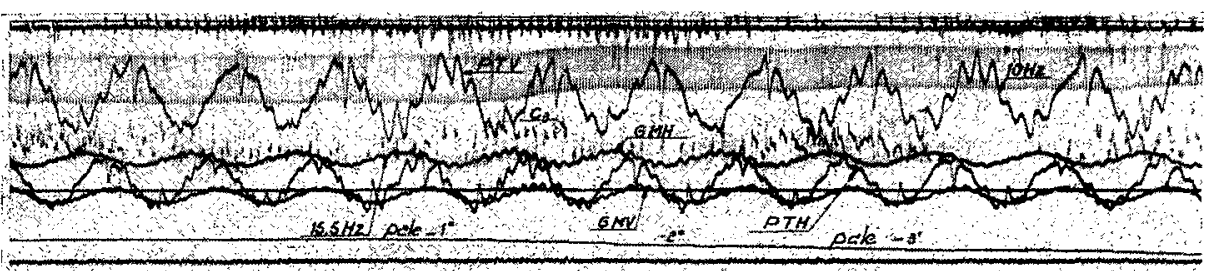

PTV : Déplacement vertical

PTH : Déplacement horizontal

GMV : Déplacement vertical

GMH : Déplacement horizontal

$C_{0}$ : Déplacement tangentiel du stator.

$\left\{\begin{array}{l}\text { Palier turbine } \\ \text { Grain mobile }\end{array}\right.$ 


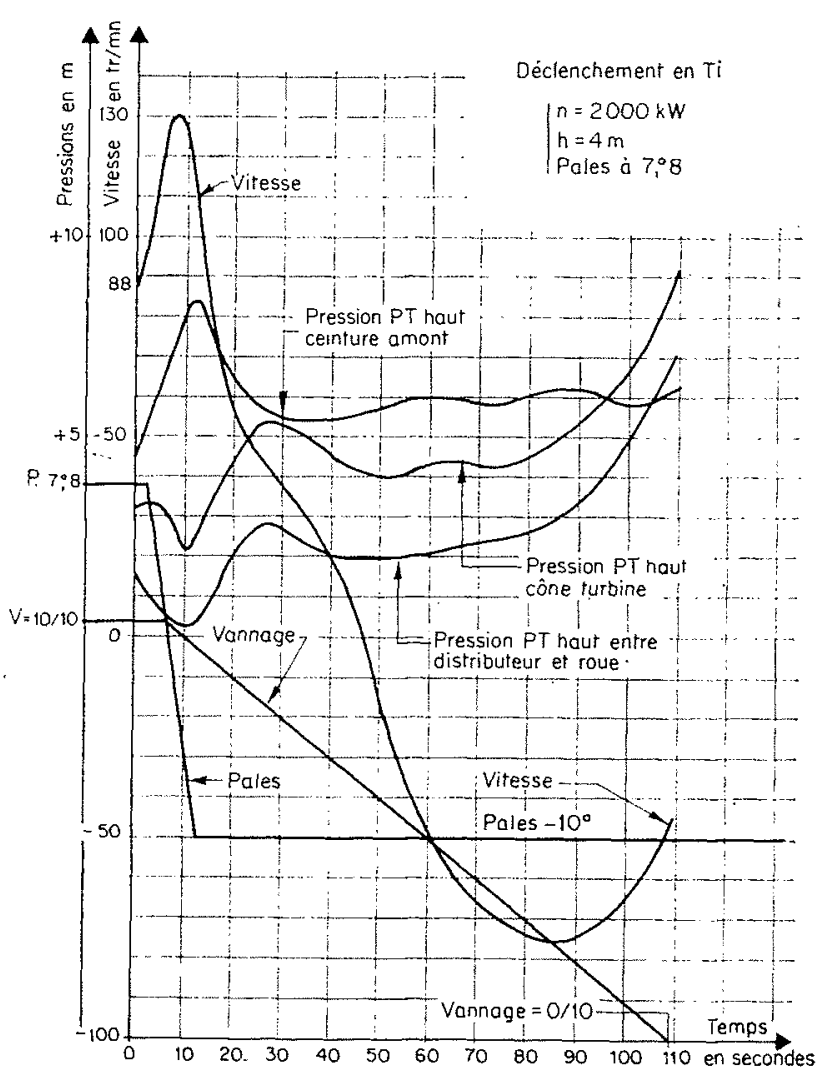

FIa. 8

- des pressions au point haut entre distributeur et roue,

- des déplacements des pales et du vannage.

Ces courbes sont tirées d'enregistrements effectués Iors d'un déclenchement en turbine inversée sous $4 \mathrm{~m}$ de chute et à la puissance de $2000 \mathrm{~kW}$ qui correspond $\dot{a}$ un angle d'inclinaison des pales de $8^{\circ}$ environ.

\section{4. Analyse des causes qui peuvent provo- quer ces vibrations de torsion. Moyens de lutter contre ces vibrations.}

Le calcul montre que la fréquence propre de l'ensemble constitué par le moulinet fixé sur l'avant-distributeur pour des mouvements autour de l'axe du groupe se situe entre 19 et $16 \mathrm{~Hz}$.

Les vibrations de torsion qui apparaissent au moment de l'application brutale du couple lors d'un démarrage en moteur asynchrone dans l'eau ou dans l'air ont une pseudo-fréquence voisine de $17 \mathrm{~Hz}$. L'amortissement de ces oscillations dans l'eau ou dans l'air étant très faible, cette fréquence correspond done à la fréquence propre de torsion du groupe.

Les vibrations de torsion d'amplitude relative- ment importante ne se produisent que lors des démarrages en moteur asynchrone et au voisinage de $73 \mathrm{tr} / \mathrm{mn}$. Ces vibrations doivent avoir pour cause la pulsation du couple asynchrone. En effet, au couple moyen d'un moteur asynchrone se superpose un couple pulsatoire dont la fréquence se définit $\operatorname{par}\left({ }^{*}\right)$ :

où :

$$
f_{p}=2 g f
$$

$g$ est le glissement en $\%$,

$f$ la fréquence du réseau, soit $50 \mathrm{~Hz}$.

Pour une vitesse synchrone de $88,3 \mathrm{tr} / \mathrm{mn}$, le glissement à $73 \mathrm{tr} / \mathrm{mn}$ est de :

$$
g=\frac{88,3-73}{88,3} \cong 17 \% \quad \text { donc } f_{p}=17 \mathrm{~Hz}
$$

Le couple pulsatoire pour la vitesse de $73 \mathrm{tr} /$ mn a une fréquence très voisine de la fréquence propre de torsion du moulinet et peut donc engendrer des vibrations de torsion dangereuses. Les amplitudes de ces oscillations seront, bien entendu, d'autant plus importantes que le temps de passage au voisinage de la vitesse de $73 \mathrm{tr} /$ mn sera long.

Nous nous devons ici de remercier MM. CarrPENTIER et RuEL, Ingénieurs aux Etablissements Alsthom, qui nous ont initiés à quelques-uns des mystères que recèlent les machines électriques et sans qui les causes de ce phénomène n'auraient pu être dévoilées.

La cause éclaircie, il reste à analyser les moyens de réduire ou de supprimer ces vibrations. Accroitre la rigidité de l'avant-distributeur de façon que la fréquence propre soit assez élevée pour qu'il n'y ait aucun risque de vibration, conduirait à une augmentation de poids considérable. Pour les groupes de la Rance la fréquence propre de torsion pourra, sans accroissement sensible du poids de l'avant-distributeur, atteindre $30 \mathrm{~Hz}$ environ. La vitesse «critique» sera donc voisine de $n=66 \mathrm{tr} / \mathrm{mn}$ pour une vitesse de synchronisme de $94 \mathrm{tr} / \mathrm{mn}$.

Les démarrages en pompe directe ou inversée peuvent s'effectuer hydrauliquement si les hauteurs de refoulement (positives ou négatives) sont suffisantes. Il a été prévu à cet effet une course angulaire négative des pales.

Ainsi, pour un démarrage hydraulique en pompe inversée, on amène le groupe au synchronisme ou à une vitesse supérieure à la vitesse critique et dans le bon sens de rotation en faisant fonctionner la machine en turbine inversée avec une inclinaison négative des pales. On couple alors sans passer, par la vitesse critique. Dans cette marche en turbine inversée, le sens de rotation est, bien entendu, opposé à celui qui

$\left(^{\star}\right)$ Consulter par exemple l'ouvrage de C. Concondia, Synchronous Machines, pp. 165 à 184 . 
correspond à la marche normale en turbine inversée.

Les passages par la vitesse critique ne se produiront done que pour les faibles hauteurs de refoulement (positives ou négatives). Dans ce cas, le couple résistant hydraulique sera réduit et le temps de passage par la zone critique court. Les amplitudes des vibrations de torsion seront donc faibles et ne compromettront pas la bonne marche de la machine. Il faut toutefois s'assurer que lors de ces démarrages, l'angle d'inclinaison des pales correspond approximativement au couple hydraulique résistant minimal. Il serait intéressant d'étudier à Saint-Malo l'influence sur ce phénomène de divers facteurs (baisse de tension, augmentation du couple hydraulique, etc.).

\section{V. - CONCLUSION}

Les nombreuses campagnes de mesures entreprises lors des premières mises en route du groupe de Saint-Malo ont montré que cette machine se prête bien à toutes les exigences des différents cycles de fonctionnement.

Les démarrages hydrauliques en pompe directe ou inversée s'effectuent sans aucune vibration au changement de flux. Les démarrages en moteur asynchrone ne présentent pas de difliculté.

Pendant les régimes de survitesses, sousvitesses et dévirages, la marche du groupe est très calme. Les passages d'un régime de fonctionnement au suivant s'effectuent sans à-coups sur les efforts de manœuvre des pales et sans vibration dangereuse pour les parties tournantes. Seules quelques vibrations de petite amplitude ont été décelées pour les faibles inclinaisons des pales qui correspondent à des zones de transition. Ces vibrations ne compromettent d'ailleurs pas le bon fonctionnement mécanique du groupe.

On peut donc envisager avec grande sûreté l'équipement à l'aide de groupes bulbes, des grandes centrales marémotrices et des installations de basses chutes. L'étude et la réalisation de machines de dimensions et de puissances plus importantes encore que celles de Saint-Malo permettront certainement de modifier l'économie des basses chutes. Les problèmes posés par l'augmentation des dimensions de ces machines concernent principalement :

- Ia construction du palier turbine, vu l'augmentation des charges,

- la conception mécanique en plusieurs pièces du stator de l'alternateur.

D I S U S I O N

président : M. Gibrat

M. le Président demande à M. Casacci de refaire passer lé premier cliché et d'indiquer sommairement, mais de façon précise, les modifications qui font partie du cahier des charges du nouveau groupe de la. Rance.

M. CASACCI donne les modifications apportées :

Le groupe de Saint-Malo est installé dans une écluse. Pour réduire les travaux de génie civil, faciliter le montage et le démontage de la machine, le conduit hydraulique autour de l'alternateur est constitué par une enveloppe métallique très rigide. L'avant-distributeur, unique support du groupe, est fixé par boulonnage sur une bride de ce conduit nuétallique. Pour les groupes «Rance», le conduit hydraulique en béton se prolonge jusqu'à l'avant-distributeur, ce dernier est boulonné sur un anneau de scellement noyé dans le béton. A la "Rance», le démontage de l'alternateur nécessite une translation horizontale, tandis qu'à Saint-Malo, le démontage de la machine s'effectue sans translation horizontale des pièces.

Pour les groupes «Rance», le problème des vibrations a fait l'objet d'études prolongées. Les fréquences propres de vibration des supports ont été augmentées. La fréquence propre de vibration de torsion de l'avant-distributeur dépasse $30 \mathrm{~Hz}$. La protection des organes supports (notamment des tirants) contre la corrosion a été particulièrement soignée.

La protection des groupes «Rance» contre les emballements prolongés en turbine inversée comporte deux stades de sécurité comme pour les Kaplan classiques. A
Saint-Malo, le distributeur n'avait pas été prévu pour fermer lors des fonctionnements en turbine inversée.

Les mesures réalisées sur le groupe de Saint-Malo et la campagne d'essais sur les efforts de manœuvre qu'effectue le Département Essais Machines de la SO.GR.E.A.H. montrent que le distributeur peut assurer en toute sécurité la fermeture des groupes lors des fonctionnements en turbine inversée. La probabilité d'une marche prolongée à l'emballement en turbine inversée est donc très faible.

La conception mécanique d'ensemble des groupes «Rance» est légèrement différente de celle de SaintMalo :

- Le servo-moteur de commande des pales est logé dans l'ogive de la roue et non entre la route et le rotor de l'alternateur;

- La butée, située entre la roue et le rotor de l'alternateur, est fixée directement sur l'avant-distributeur. A Saint-Malo elle est logée dans le croisillon de l'alternateur, côté calotte de réfrigération.

M. le Président remercie M. CASACCI el ouvre la discussion.

M. Cazenave demande si M. Casacci peut donner des précisions sur les démontages, notamment ceux qui sont utiles en exploitation pour des interventions courantes.

M. CaSACCr dit que pour les groupes «Rance 》, à l'iniliative d'E.D.F., toutes les dispositions ont été prises pour que l'entretien des groupes soit comparable à celui d'une Kaplan verticale classique. 
1. MONTAGE ET DÉMONTAGE POUR ASSURER L'ENTRETIEN DU GROUPE.

1.1 Partie mécanique.

Peuvent se demonter et remonter saus manutention importante :

- A travers les bras du rotor de l'alternateur :

- le coussinet du palier turbine,

- le joint turbine,

- les patins de la butée double.

- Par le bras d'accès:

- le coussinet du palier alternateur,

- les alvéoles de distribution d'huile sous pression au servomoteur de commande des pales,

- les divers auxiliaires de graissage, récupération des fuites, etc.

\section{2 Partie électrique.}

En ce qui concerne l'alternateur, il est possible de dérnonter sans manutention importante :

- un ou plusieurs pôles du rotor,

- des jeux de barres.

Il est d'autre part possible de rebobiner complètement le stator et de réparer un rotor très gravement accidenté en effectuant un démontage partiel du groupe:

- démontage du manteau de la roue,

- déboulonnage de l'avant-distributeur sur son anneau de scellement et de la carcasse sur l'avant-distributeur.

On opère alors la translation de l'ensemble constitué par le distributeur, l'avant-distributeur et les parties tournantes, la roue pénétrant dans l'aspirateur. Pour effectuer cette translation, le groupe est supporté d'une part par un chariot mobile et guidé d'autre part par un faux arbre qui glisse dans le palier alternateur, lequel est resté fixe puisqu'il est solidaire de l'anneau à tirants. Le groupe est ensuite fixé sur l'anneau de scellement à l'entrée de l'aspirateur, la roue se trouvant dans l'aspirateur.

Après cette translation, il est possible d'effectuer le rebobinage complet du stator et, si nécessaire, soit la réparation du rotor, soit son démontage et son remplacement.

Seuls des accidents très graves au rotor ou au stator de l'alternateur nécessiteront un tel démontage.

2. Montage ET DéMontage DU GROUPE.

Les principales opérations de montage du groupe sont les suivantes :

- Mise en place de l'ogive et du bras d'accès dans le conduit hydraulique;

- Descente de l'ensemble rotor-stator de l'alternateur;

- Translation de l'ensemble rotor-stator de l'alternateur que l'on glisse dans le conduit hydraulique à l'aide d'un chariot;

- Fixation de la carcasse de l'alternateur sur l'anneau à tirants;

- Mise en place et fixation de l'avant-distributeur et du distributeur;

- Descente, mise en place et fixation du moulinet comportant la roue sans les pales, le palier et l'arbre turbine, le cone support de palier;

- Montage des pales;

- Montage du manteau de la roue.

Sur une question de M. Ménard, M. CaSAGGi précise que le démontage $d u$ coussinet $d u$ palier turbine s'effectue a travers les bras du rotor de l'alternateur. Cetle opération a été réalisée à Saint-Malo.

M. Darnieus signale qu'il y a quinze ans, pour la soufflerie d'Hispano-Suiza, dont le ventilateur a 8 mètres de diamètre, on s'était trouvé en présence d'un problème du méme genre et, pour éviter la rotation autour de l'axe, on avait incliné les aubes directrices sur le rayon dans le plan perpendiculaire à l'axe. C'est une méthode employée pour des turbines à gaz et, gènéralement, là où il $\mathrm{y}$ a de grandes différences de température.

M. Casaccr remercie M. Darrieus pour ces précisions intéressantes et signale que l'on avait pensé à prévoir des tirants tangentiels pour augmenter la rigidité de torsion $d u$ groupe. On aurait facilement pu obtenir des fréquences propres de 50 à $60 \mathrm{~Hz}$ à l'aide de tirants tangentiels.

M. Darrieus ajoute que les aubes directrices sont assez nombreuses et l'on obtient assez facilement ce résultat avec une faible inclinaison.

M. Casacci convient qu'il est effectivement possible d'augmenter la rigidité de torsion de l'avant-distributeur en inclinant légèrement les avant-directrices. Les différents régimes de fonctionnement des groupes marémoteurs (double flux) rendent difficile l'adoption de cette disposition sans détérioration des performances.

M. Narcy rappelle qu'en ce qui concerne les vibrations de torsion, il $\mathrm{y}$ a un régime troublé à prendre en considération : la marche au moment d'un décrochage de l'alternateur. A ce moment-là, avec la pulsation, on peut avoir un couple asynchrone et qui change de signe : il est positif et négatif à la fréquence du battement.

M. Casacar signale qu'à Saint-Malo de nombreux essais de déclenchement ont été réalisés et qu'aucune vibration nuisible $n^{\prime a}$ été constatée.

M. Narcy dit que ce n'est pas ce régime qu'il faut considérer, mais celui de l'alternateur en asynchrone avec la superposition du champ rotor et du champ stator, qui n'a pas la même fréquence. Cette période doit être assez courte, mais elle est tout de même à prendre en considération.

M. Casacci précise que les électriciens du groupe 《Rance» examineront tous les cas de fonctionnement qui peuvent engendrer des oscillations de torsion dans l'avanl-distributeur et jemercie M. Nancy pour ses rematques.

M. Vazélle rappelle qu'au début de son exposé, M. Casaccr a attiré l'attention sur l'importance qu'il fallait attacher à la bonne alimentation du conduit hydraulique. La présence du bras vertical d'accès dans le bulbe 1ue présente-t-il pas d'inconvénients à cet égard et ne serait-il pas préférable de prévoir un second bras support sous la calotte pour rétablir la symétrie de l'ensemble?

M. Casacar précise que le hras d'accès est déjà loin du distributeur. La perturbation de l'éconlement dans le distributeur, due à la présence du bras d'accès, ne doit donc pas être très importante. L'adjonction d'un support vertical à la partie inférieure de l'ogive, situé dans le prolongement du bras d'accès, rendrait effectivement l'écoulement plus symétrique, mais réduirait de facon sensible la section du conduit, ce qui entraînerait très probablement une réduction de performance.

M. CAzENAvE connait des projets dans lesquels on a prévu un accès horizontal du bulbe permettant le passage courant du personnel d'exploitation. Au surplus, d'autres obstacles sont prévus dans le conduit amont pour l'appui du bulbe, l'évacuation des câbles. A-t-on déterminé avec précision Jes dimensions maximales qui peuvent être données à ces obstacles sans altérer les performances?

M. Casaccr précise qu'au Département Essais Machines dirigé par M. Duport, toute une série d'essais a été réalisèe pour étudier l'influence sur les performances de la présence d'obstacles (bras d'accès, support), situés dans le conduit hydranlique. Pour des machines ne fonc. tionnant qu'en turbine directe, les sillages dus à la présence des bras d'accès ou des supports ont peu d'influence sur les performances si ces obstacles placés dans le conduit sont assez éloignés du distributeur.

M. le Président remercie M. Casaccr. 